\title{
Autotrasplante (AT) de progenitores hematopoyéticos en mieloma múltiple. Experiencia clínica
}

\author{
Claudio Flores P, G uillermo Conte L, Patricia Fardella B, \\ Daniel Araos H, Jorge Alfaro L, Paola Aravena R, \\ Néstor G onzález G, Milton Larrondo L.
}

\section{Autologous transplant (AT) with peripheral-blood stem-cell rescue for multiple myeloma. A clinical experience}

Background: Multiple myeloma is rarely curable. Advances in high dose chemotherapy and stem cell transplantation have improved overall survival and event-free disease periods, but relapses are inevitable. Aim: To report our experience with AT in multiple myeloma, between 1994 and 2003. Material and Methods: Retrospective analysis of 20 patients (12 women), with a mean age of 51.1 years. VAD (vincristine, doxorubicin and dexamethasone) was used as initial therapy in 19 patients. High dose cyclophosphamide (11 patients) and variations of VAD regimen (7) associated with granulocyte colony stimulating factor were used for peripheral-blood stem cell harvest. The conditioning regimen consisted of melphalan $200 \mathrm{mg} / \mathrm{m}^{2}$ followed by the reinfusion of peripheral-blood stem cells 24 hours later. The median number of CD34 cells infused was $3,3 \times 10^{6} / \mathrm{kg}$. Three patients were subjected to a second auto graft and one to a non-myeloablative transplant. Mean follow up was 35.5 months. Results: Mucositis and febrile neutropenia were common complications. The median number of days for neutrophyl engraftment was 9 (range 8-11) and for platelets, 10 (range 7-13). No patient died. Complete remission was obtained in $60 \%$ (12/20), progession-free survival was 30 months and overall median survival, 47 months. Conclusions: The AT with high-dose melphalan is a safe procedure in our hospital, without mortality and engraftment in all the patients. Complete remission and progression free survival were similar to those reported abroad but the overall median survival was lower (Rev Méd Chile 2005; 133: 887-93).

(Key Words: Hematopoietic stem cells; Melphalan; Multiple myeloma)

Recibido el 7 de octubre, 2004. Aceptado en versión corregida el 12 de abril, 2005.

Sección Hematología y Banco de Sangre, Hospital Clínico Universidad de Chile.

$E^{1}$ mieloma múltiple continúa siendo una enfermedad incurable con las terapias disponibles

Correspondencia a: Dr. Claudio Flores P. Hospital Clínico de la Universidad de Chile, Santos Dumont 999, Santiago, Chile. Fax: (562) 6788248. E mail: cflores@redclinicauchile.cl actualmente ${ }^{1}$. Un avance notable en los últimos años ha sido la introducción de la quimioterapia en dosis altas con rescate con progenitores hematopoyéticos autólogos, o autotrasplante (AT), el cual ha demostrado ser superior a los esquemas convencionales en estudios comparativos, con 
mayor proporción de respuestas completas y una sobrevida global media de 54-56 meses, la cual es superior en 12 meses a la obtenida con quimioterapia estándar2,3. El objetivo de este trabajo es mostrar nuestros resultados de nuestro centro en 20 pacientes portadores de mieloma múltiple seleccionados para AT.

\section{Material y Métodos}

Se evaluaron 20 pacientes con diagnóstico de mieloma múltiple, que fueron tratados con trasplante autólogo de médula ósea en la Sección de Hematología del Hospital Clínico de la Universidad de Chile, en el período comprendido entre junio de 1994 y julio de 2003.

Tabla 1. C aracterísticas clínicas previo al trasplante

\begin{tabular}{|c|c|c|c|c|c|}
\hline Pacientes & $\begin{array}{l}\text { Edad } \\
\text { (años) }\end{array}$ & Sexo & Tipo de mieloma & Estadio & $\begin{array}{c}\text { Terapia previo a TAMO } \\
\left.\text { ( } \mathrm{N}^{\circ} \text { de ciclos }\right)\end{array}$ \\
\hline 1 & 62 & $\mathrm{~F}$ & IgG Lambda & III A & $\begin{array}{l}\text { Melfalán Prednisona (3) } \\
\text { Radioterapia } \\
\text { Metilprednisolona (6) } \\
\text { VAD (4) }\end{array}$ \\
\hline 2 & 37 & $\mathrm{~F}$ & CL Lambda & III B & VAD (4) \\
\hline 3 & 61 & $\mathrm{M}$ & IgG Карра & III A & VAD (3) \\
\hline 4 & 42 & $\mathrm{M}$ & IgG Lambda & III A & VAD (4) \\
\hline 5 & 57 & $\mathrm{~F}$ & IgG Kappa & III A & $\begin{array}{l}\text { Melfalán Prednisona (5) } \\
\text { Dexametasona (3) } \\
\text { VAD (2) }\end{array}$ \\
\hline 6 & 51 & $\mathrm{~F}$ & CL Lambda & III A & VAD (3) \\
\hline 7 & 48 & $\mathrm{~F}$ & CL Lambda & III A & VAD (3) \\
\hline 8 & 39 & $\mathrm{~F}$ & IgG Карра & III B & $\begin{array}{l}\text { VAD (3) } \\
\text { Dexametasona (1) }\end{array}$ \\
\hline 9 & 53 & M & IgA Kappa & III A & $\begin{array}{l}\text { VAD (4) } \\
\text { ESHAP (2) }\end{array}$ \\
\hline 10 & 52 & $\mathrm{~F}$ & IgG Kappa & III A & VAD (6) \\
\hline 11 & 55 & $\mathrm{~F}$ & IgA Lambda & III A & $\begin{array}{l}\text { Melfalán Prednisona (1) } \\
\text { VAD (4) }\end{array}$ \\
\hline 12 & 43 & M & No secretor & III A & $\mathrm{CHOP}(6)$ \\
\hline 13 & 53 & M & IgG Lambda & II A & VAD (6) \\
\hline 14 & 55 & M & IgG Kappa & III A & $\begin{array}{l}\text { VAD (3) } \\
\text { Radioterapia } \\
\text { CVAD (1) }\end{array}$ \\
\hline 15 & 56 & $\mathrm{~F}$ & IgA Карра & III A & VAD (3) \\
\hline 16 & 60 & $\mathrm{~F}$ & IgG Kappa & III $\mathrm{A}$ & VAD (5) \\
\hline 17 & 47 & M & IgG Kappa & III A & $\begin{array}{l}\text { Melfalán Prednisona (1) } \\
\text { VAD (3) } \\
\text { Metilprednisolona }\end{array}$ \\
\hline 18 & 44 & $\mathrm{~F}$ & CL Lambda & III B & VAD (3) \\
\hline 19 & 58 & $\mathrm{~F}$ & IgA Lambda & II A & VAD (3) \\
\hline 20 & 49 & M & IgA Kappa & III A & $\begin{array}{l}\text { Melfalán Prednisona (2) } \\
\text { VAD (4) }\end{array}$ \\
\hline
\end{tabular}

CL: Cadenas ligeras. 
La indicación de trasplante en estos pacientes se basa en que con este procedimiento se obtiene una mayor tasa de respuestas completas y una mayor sobrevida global media que con la quimioterapia convencional. En todos ellos se realizó un estudio pre-trasplante para evaluar sus condiciones generales previo al procedimiento.

Se estudiaron 12 mujeres y 8 hombres, la edad promedio del grupo fue 51,1 años (rango 37-62 años). Según tipo de mieloma, los pacientes se distribuían de la siguiente forma: 10 pacientes IgG (7 Kappa y 3 Lambda), 5 IgA (3 Kappa y 2 Lambda), 4 cadenas ligeras Lambda y un paciente con mieloma no secretor. La etapa clínica al diagnóstico, según la clasificación de Durie Salmon era: 18 pacientes en etapa III y 2 en etapa II. Tres pacientes tenían creatinina $>2 \mathrm{mg} / \mathrm{dL}, 2$ de ellos portadores de mieloma de cadenas ligeras. El tratamiento previo al autotrasplante consistió en quimioterapia con esquema VAD en 19 pacientes, en un número de 3 a 4 ciclos (vincristina $0,4 \mathrm{mg} /$ día $\mathrm{x} 4$ días y doxorubicina $9 \mathrm{mg} / \mathrm{m}^{2} /$ día x 4 días, ambas en infusión continua, dexametasona $40 \mathrm{mg} /$ día, los días 1-4, 9-12 y 17-20 del ciclo) administrada cada 28 días. Cinco pacientes recibieron, además, entre 1 y 5 ciclos de melfalán y prednisona; estos pacientes fueron tratados en otros centros antes de ser referidos a este hospital para continuar con su terapia. Las características de los pacientes se muestran en la Tabla 1.

El tiempo promedio entre el diagnóstico y el autotrasplante fue de 8,5 meses (rango 5-17 meses). Los pacientes se evaluaron después de recibir el $3^{\text {er }}$ o $4^{\circ}$ ciclo de $\mathrm{VAD}$, con electroforesis de proteínas del plasma (EFP), mielograma, cuantificación de inmunoglobulinas y proteinuria de $24 \mathrm{~h}$ para determinar la quimiosensibilidad previa al trasplante. Según estos parámetros, encontramos 16 pacientes quimiosensibles, presentando una respuesta mayor de 50\%; un paciente no tuvo respuesta clínica y 3 evolucionaron con enfermedad progresiva durante la quimioterapia luego de haber presentado una respuesta parcial transitoria (Tabla 2). La movilización de progenitores hematopoyéticos de sangre periférica se realizó con ciclofosfamida, en dosis 3 $\mathrm{g} / \mathrm{m}^{2}\left(1,5-4 \mathrm{~g} / \mathrm{m}^{2}\right)$ en 11 pacientes; C-VAD (ciclofosfamida $300 \mathrm{mg} / \mathrm{m}^{2} \mathrm{c} / 12 \mathrm{~h}$ x 6 dosis vía intravenosa (iv); vincristina $2 \mathrm{mg}$ iv día 4; doxorubicina $50 \mathrm{mg} /$ $\mathrm{m}^{2}$ iv día 4 y dexametasona $40 \mathrm{mg} /$ día $\mathrm{x} 4$ días vía oral) en 5 pacientes; VAD en 2 casos y otros esquemas en los 2 restantes (Tabla 2).

Tabla 2. Q uimiosensibilidad y esquema de movilización

\begin{tabular}{|cccc|}
\hline Pacientes & $\begin{array}{c}\text { Tiempo previo a } \\
\text { TAMO (meses) }\end{array}$ & $\begin{array}{c}\text { Respuesta a } \\
\text { terapia previa }\end{array}$ & $\begin{array}{c}\text { Esquema de } \\
\text { movilización }\end{array}$ \\
\hline 1 & 12 & Quimiosensible & ESHAP \\
2 & 8 & Quimiosensible & VAD \\
3 & 12 & Quimiosensible & Ciclofosfamida \\
4 & 17 & Quimiosensible & Ciclofosfamida \\
5 & 15 & Quimiosensible & CVAD \\
6 & 6 & Quimiosensible & Ciclofosfamida \\
7 & 6 & Quimiosensible & CVAD \\
8 & 8 & Quimiosensible & Ciclofosfamida \\
9 & 8 & Refractario & ESHAP \\
10 & 14 & Quimiosensible & Ciclofosfamida \\
11 & 5 & Quimiosensible & VAD \\
12 & 8 & Quimiosensible & CVAD \\
13 & 13 & Quimiosensible & Ciclofosfamida \\
14 & 5 & Quimiosensible & CVAD \\
15 & 5 & Quimiosensible & CVAD \\
16 & 14 & Progresión & Ciclofosfamida \\
17 & 11 & Quimiosensible & Ciclofosfamida \\
18 & 5 & Quimiosensible & Ciclofosfamida \\
19 & 9 & Progresión & Ciclofosfamida \\
20 & 10 & & \\
\hline
\end{tabular}


En todos los pacientes se asoció factor estimulante de colonias (G-CSF), en dosis de $300 \mu \mathrm{g} /$ día vía subcutánea, a partir del día +8 de la quimioterapia. La dosis de G-CSF se aumentó al doble el día previo a la recolección y los recuentos de CD34 se realizaron a partir del día +12 . La recolección de progenitores hematopoyéticos se efectuó a través de un catéter 7 French, en una máquina separadora de células Cobe Spectra.

El condicionamiento del trasplante se realizó con melfalán en dosis total de $200 \mathrm{mg} / \mathrm{m}^{2}$, administrado los días -3 y -4, y dexametasona 100 $\mathrm{mg}$ iv los mismos días, que es uno de los condicionamientos estándar en AT. La infusión de progenitores se realizó 2 días después en una dosis promedio de $3,3 \times 10^{6} \mathrm{CD} 34 / \mathrm{kg}$. Se trasplantaron 2 pacientes con creatinina entre 3 y $4 \mathrm{mg} /$ dL, sin ajuste de dosis de melfalán. La respuesta al trasplante se evaluó a los 3 meses con: mielograma, EFP, cuantificación de inmunoglobulinas y proteinuria de $24 \mathrm{~h}$. Se utilizó terapia de mantención post AT en 8 pacientes; en 2 pacientes se usó Interferón alfa 2a y en 6 la asociación de talidomida y dexametasona. El seguimiento promedio del grupo fue de 35,5 meses (rango: 21-90 meses).

Estadística: Promedios y desviaciones estándar. Curvas de sobrevida de Kaplan Meier. Se determinó la sobrevida global, que corresponde al tiempo entre el inicio de la terapia citorreductora, VAD u otra, hasta la muerte por cualquier causa y la sobrevida libre de progresión (SLP) que se define como el tiempo transcurrido entre el trasplante y evidencia de progresión en uno o más de los siguientes parámetros: aumento de paraproteína en suero o en orina, aparición de nueva lesión osteolítica, aumento de nivel de infiltración plasmocelular en médula.

\section{Resultados}

De los 20 pacientes trasplantados, en todos se produjo prendimiento del trasplante. La recuperación de neutrófilos (> 500/ $\mu \mathrm{L}$ ) fue al día 9 (rango 8-11 días) y la de plaquetas (> 20.000/ $\mu \mathrm{L}$ e independencia de transfusión plaquetaria) el día 10 (rango 7-13 días). En cuanto a las complicaciones no infecciosas, la mucositis fue la más frecuente y se observó en 19/20 pacientes, con compromiso orofaríngeo e intestinal. Presentaron mucositis grado 3-4 (OMS), 15 pacientes y grado 1-2 (OMS), 4. Para el manejo de este cuadro se utilizó analgesia opiácea, soporte nutricional parenteral y medidas locales.

Observamos trombosis venosa en 3 pacientes (15\%) durante todo el proceso de trasplante; un caso de trombosis venosa profunda distal durante quimioterapia $\mathrm{VAD}$, un episodio de tromboembolismo pulmonar después de movilización de progenitores hematopoyéticos con ciclofosfamida; y un episodio de trombosis venosa del tronco braquiocefálico durante el trasplante, asociada a presencia de catéter venoso central instalado por yugular interna izquierda. Todos estos episodios se trataron con heparina de bajo peso molecular y luego, anticoagulantes orales. Observamos toxicidad hepática grado III transitoria con encefalopatía en un paciente, cuya evaluación previa al trasplante no mostraba signos de patología de este órgano.

De los dos pacientes que cursaron con insuficiencia renal, en ninguno se observó aumento de sus niveles de creatinina durante el procedimiento; en uno de ellos se documentó mejoría de la función renal. En cuanto a las complicaciones infecciosas, el $90 \%$ de los pacientes presentó fiebre durante el perÍodo de neutropenia y en $30 \%$ de los episodios febriles se demostró bacteremia. Los gérmenes aislados fueron $\mathrm{E}$ coli (3 pacientes), Saureus meticilino sensible (1 paciente), enterococo (1 paciente) y S Beta hemolítico (1 paciente). Una paciente presentó el día de la reinfusión de progenitores hematopoyéticos una peritonitis bacteriana espontánea grave, asociada a shock séptico y falla respiratoria catastrófica, por lo que necesitó hospitalización y manejo en la Unidad de Tratamiento Intensivo 4 .

El período de hospitalización fue 23 días (1465 días). En cuanto a la respuesta al trasplante, ella fue evaluada al $3^{\text {er }}$ mes del procedimiento. Se observó respuesta completa, con recuento de plasmocitos en médula inferior a 5\%, desaparición de peak monoclonal en electroforesis sérica y ausencia de proteinuria, en 12 pacientes (60\%). En 2 de ellos se documenta inmunofijación negativa. En 8 pacientes se obtuvo respuesta parcial y en 1 paciente no se observa respuesta objetiva con el trasplante, progresando la enfermedad a los pocos meses. 
Tabla 3. Evolución y respuesta al trasplante

\begin{tabular}{|ccccccc|}
\hline Pacientes & $\begin{array}{c}\text { Prendimiento } \\
\text { neutrófilos } \\
\text { (días) }\end{array}$ & $\begin{array}{c}\text { Prendimiento } \\
\text { plaquetas } \\
\text { (días) }\end{array}$ & $\begin{array}{c}\text { Respuesta al } \\
\text { trasplante }\end{array}$ & $\begin{array}{c}\text { Sobrevida } \\
\text { global } \\
\text { (meses) }\end{array}$ & $\begin{array}{c}\text { Sobrevida } \\
\text { ibre eventos } \\
\text { (meses) }\end{array}$ & $\begin{array}{c}\text { Estado del } \\
\text { paciente }\end{array}$ \\
\hline 1 & 9 & 10 & $\mathrm{RC}$ & 90 & 64 & $\mathrm{~F}$ \\
2 & 10 & 13 & $\mathrm{RC}$ & 27 & 11 & $\mathrm{~F}$ \\
3 & 9 & 10 & $\mathrm{RP}$ & 61 & 46 & $\mathrm{~F}$ \\
4 & 8 & 9 & $\mathrm{RP}$ & 47 & 14 & $\mathrm{~F}$ \\
5 & 11 & $\mathrm{ND}$ & $\mathrm{RP}$ & 23 & 18 & $\mathrm{~F}$ \\
6 & 9 & 10 & $\mathrm{RC}$ & 43 & 30 & $\mathrm{~V}-\mathrm{PE}$ \\
7 & 8 & 7 & $\mathrm{RC}$ & 50 & 35 & $\mathrm{~V}-\mathrm{PE}$ \\
8 & 9 & 11 & $\mathrm{RC}$ & 49 & 41 & $\mathrm{~V}$ \\
9 & 9 & 8 & $\mathrm{Nula}$ & 42 & 0 & $\mathrm{~F}$ \\
10 & 9 & 9 & $\mathrm{RC}$ & 39 & 9 & $\mathrm{~F}$ \\
11 & 10 & 8 & $\mathrm{RC}$ & 29 & 12 & $\mathrm{~V}-\mathrm{PE}$ \\
12 & 9 & 8 & $\mathrm{RC}$ & 39 & 12 & $\mathrm{~F}$ \\
13 & 8 & 6 & $\mathrm{RC}$ & 38 & 25 & $\mathrm{~V}$ \\
14 & 8 & 10 & $\mathrm{RP}$ & 31 & 15 & $\mathrm{~V}$ \\
15 & 8 & 9 & $\mathrm{RC}$ & 26 & 21 & $\mathrm{~V}$ \\
16 & 8 & 8 & $\mathrm{RC}$ & 33 & 19 & $\mathrm{~V}$ \\
17 & 9 & 10 & $\mathrm{RP}$ & 28 & 17 & $\mathrm{~V}$ \\
18 & 10 & 10 & $\mathrm{RC}$ & 21 & 16 & $\mathrm{~V}$ \\
19 & 9 & 10 & $\mathrm{RP}$ & 23 & 14 & $\mathrm{~V}$ \\
20 & 11 & 10 & $\mathrm{RP}$ & 23 & 7 & $\mathrm{~V}-\mathrm{PE}$ \\
\hline
\end{tabular}

RP: Respuesta parcial. RC: Respuesta completa. F: Fallecido. V: Vivo, enfermedad estable. V-PE: Vivo, progresión de su enfermedad.

En 3 pacientes se realizó un segundo trasplante autólogo con melfalán $200 \mathrm{mg} / \mathrm{m}^{2}$, dos de estos pacientes se encontraban en remisión completa luego del primer AT y el tercero no presentó ninguna respuesta luego de realizar ambos procedimientos. Una paciente que obtuvo respuesta completa luego de AT se sometió a un trasplante alogénico con condicionamiento de intensidad reducida, al momento de detectar progresión de su mieloma y luego de 1 año de seguimiento. Con este último procedimiento alcanzó respuesta parcial. La sobrevida libre de progresión actuarial media del grupo fue de 30 meses. Se incluyó en el análisis al paciente que no obtuvo respuesta, con una SLP nula; la sobrevida global estimada fue 47 meses.

No hubo muertes durante el procedimiento de autotrasplante ni dentro de los primeros 6 meses posteriores; durante el seguimiento fallecieron 8 pacientes, todos en recaída, presentando enfermedad refractaria.

\section{Discusión}

La quimioterapia en dosis altas con rescate de progenitores autólogos se ha constituido en la forma de tratamiento estándar de pacientes con mieloma múltiple menores de 65 años, en condición fisiológica adecuada. La sobrevida comunicada es superior a la obtenida con esquemas de quimioterapias convencionales. La quimioterapia inicial de pacientes con mieloma recién diagnosticados, potencialmente candidatos a AT, debe ser con esquemas que no contengan drogas que afecten la reserva de células progenitoras hemato- 
poyéticas, como melfalán y nitrosoureas ${ }^{5}$. El esquema melfalán-prednisona cuando es utilizado en estos pacientes puede hacer muy difícil 0 imposible obtener el número adecuado de progenitores hematopoyéticos para realizar el procedimiento de AT. En nuestra serie 5 pacientes fueron tratados con melfalán-prednisona antes de ser derivados a nuestro centro, recibiendo entre 1 y 5 ciclos; sin embargo en todos ellos logró cosecharse un número suficiente de células CD34 para el AT. El esquema VAD se utiliza como tratamiento inicial, pues no afecta en forma significativa la probabilidad de obtener una cosecha exitosa de progenitores hematopoyéticos.

El condicionamiento clásicamente se hace con melfalán endovenoso, $200 \mathrm{mg} / \mathrm{m}^{2}$, sin necesidad de ajustar dosis en insuficiencia renal. Se han utilizado otros esquemas incluyendo combinaciones con otras drogas citotóxicas y con radioterapia corporal total, pero no se han demostrado superiores a melfalán solo ${ }^{1,6-8}$. Sigue siendo discutible si los resultados son mejores con doble trasplante ${ }^{9}$ y también si es necesario demostrar quimiosensibilidad antes de ofrecer esta modalidad terapéutica. Una paciente en esta serie, con enfermedad progresiva e insuficiencia renal, alcanza respuesta completa luego del AT, con inmunofijación negativa, con 3 años de seguimiento hasta esta fecha.

En nuestro grupo la sobreviva global estimada es de 47 meses, y la sobrevida media libre de progresión de 30 meses. Estas cifras son algo inferiores a lo reportado en estudios multicéntri$\cos ^{2,3}$; esta diferencia puede ser real, o bien relacionada al pequeño número de pacientes tratados en esta serie. Por otro lado, es difícil comparar resultados ya que no existe un índice de riesgo pronóstico que permita homologar diferentes grupos de pacientes. Los factores pronósticos clásicos son: etapa clínica, elevación de creatinina, de $§ 2$ microglobulina, lactato deshidrogenasa y proteína $\mathrm{C}$ reactiva; edad avanzada y grado de respuesta a tratamiento o quimiosensibilidad; por otra parte los criterios pronósticos de mayor peso, aunque no siempre al alcance del clínico, son el índice proliferativo y las alteraciones citogenéticas de las células tumorales ${ }^{1,10,11}$. En nuestro grupo no disponemos de beta 2 microglobulina sérica en todos nuestros pacientes al momento del diagnós- tico (varios fueron referidos para trasplante desde otros centros) y tampoco estudiamos en forma rutinaria las alteraciones citogenéticas ni el índice proliferativo, por lo que se hace necesario para nuestro próximo grupo de candidatos a trasplante, la incorporación de estos elementos para una mejor evaluación de nuestros resultados.

Se utilizó tratamiento de mantención sólo en algunos casos. El Interferón fue mal tolerado en general por sus efectos adversos clásicos, incluyendo citopenias. Debido a reportes sobre neoformación vascular en la médula ósea que precede a la reactivación o progresión del mieloma múltiple tratado, que puede ser bloqueada con talidomi$\mathrm{da}^{12,13}$, decidimos probar su efecto como mantención luego de trasplante en dosis de $100 \mathrm{mg} /$ día, sola o asociada a esteroides. Hasta ahora los datos no permiten sacar conclusiones respecto a la eficacia de esta medida, pero es notoria la neurotoxicidad que produce en la mayoría de los pacientes, que sin ser grave puede afectar la calidad de vida.

En la actualidad se estudian otras alternativas terapéuticas. El trasplante alogénico con condicionamiento de intensidad reducida o no mieloablativo está en evaluación, pero el efecto de injerto versus enfermedad no ha sido tan importante como el que se observa por ejemplo en linfomas de bajo grado y leucemia mieloide crónica, lo que podría condicionar una menor respuesta en este grupo de pacientes. Una alternativa promisoria recientemente incorporada es el Bortezomib (PS 341), que actúa modulando la expresión de factores de transcripción críticos, de genes supresores de tumores y de otras proteínas reguladoras del ciclo celular en las células de mieloma. El Bortezomib se ha demostrado muy activo en pacientes con mieloma, incluso en pacientes refractarios a quimioterapia ${ }^{14,15}$ y su uso en combinación con otras modalidades terapéuticas parece mejorar la respuesta clínica.

En base a los resultados expuestos podemos señalar que en nuestro centro el AT es un procedimiento factible y seguro en pacientes con mieloma múltiple, resultando notable que pese a la significativa morbilidad asociada, la recuperación hematológica fue rápida y no hubo mortalidad en nuestro grupo. 


\section{REFERENCIAS}

1. Dispenzieri A, Lacy M, GreipP P. Capítulo «Multiple Myeloma»en Wintrobe's Clinical Hematology, $11^{\text {th }}$ Edition, Lippincot Williams and Wilkins, 2004.

2. Attal M, Harousseau JL, Stoppa AM, Sotto JJ, FuziBet JG, Rossi JF et AL. A prospective, randomized trial of autologous bone marrow transplantation and chemotherapy in multiple myeloma. $\mathrm{N}$ Engl J Med 1996; 335: 91-7.

3. Child Ja, Morgan G, Davies F, Faith D, Ower R, BELL S ET AL. High-dose chemotherapy with hematopoietic stem-cell rescue for multiple myeloma. N Engl J Med 2003; 348: 1875-83.

4. Conte G, Flores C, Fardella P, Araos D, Alfaro J, Barahona K et al. Primary peritonitis (E coli) as a complication of autologous bone marrow transplantation in multiple myeloma. Blood 1995; 86: 942a.

5. Tricot G, Jagannath S, Vesole D, Nelson J, Tindle S, Miler L ET AL. Peripheral blood stem cell transplants for multiple myeloma: identification of favorable variables for rapid engrafment in 225 patients. Blood 1995; 85: 588-96.

6. Moreau P, Facon T, Attal M, Huln C, Michadet M, MaLoissel F et al. Comparison of $200 \mathrm{mg} / \mathrm{m}^{2}$ melphalan and $8 \mathrm{~Gy}$ total body irradiation plus $140 \mathrm{mg} / \mathrm{m}^{2}$ melphalan as conditioning regimens for peripheral blood stem cell transplantation in patients with newly diagnosed multiple myeloma. Blood 2002; 99: 731-5.

7. Einsele $H$, Bamberg M, Budach W, Schmidberger $H$, Hess CF, Worman B et al. A new conditioning regimen involving total marrow irradiation, busulfan and cyclophosphamide followed by autologous PBSCT in patients with advanced multiple myeloma. Bone Marrow Transplant 2003; 32: 593-9.

8. Cogie CR, Moreb JS, Leather HL, Finiewicz KJ, Khan SA, REDDY VS ET AL. Busulfan, cyclophosphamide and etoposide as conditioning for autologous stem cell transplantation in multiple myeloma. Am J Hematol 2003; 73: 169-75.

9. Fassas AB, Barlogie B, Ward S, Jagannath S, Vesole D, Matтох S Eт AL. Survival after relapse following tandem autotransplant in multiple myeloma: the University of Arkansas total therapy I experience. Br J Haematol 2003; 123: 484-9.

10. BJorkstrand B, Goldstone AH, Ljungman P, BRandt L, Brunet S, Carlso K et al. Prognostic factors in autologous stem cell transplantation for multiple myeloma: an EBMT Registry Study. Leuk Lymphoma 1994; 15: 265-72.

11. Rajkumar SV, Fonseca R, Lacy MQ, Witzig T, Lust J, GREIPP P ET AL. Beta 2 microglobulin and bone marrow plasma cell involvement predict complete responders among patients undergoing blood cell transplantation for myeloma. Bone Marrow Transplant 1999; 23: 1261-6.

12. Folkman J. Angiogenesis-Dependent Diseases. Seminars in Oncology 2001; 28: 536-42.

13. D'Amato R, Lentzsch S, Anderson K, Rogers M. Mechanism of action of Thalidomide and 3Aminothalidomide in Multiple Myeloma. Seminars in Oncology 2001; 28: 597-601.

14. Adams J. Proteasome Inhibition in Cancer: Development of PS-341. Seminars in Oncology 2001; 28; 613-9.

15. Richardson P, Barlogie B, Berenson J, Singhal S, Jagannath S, Irwin W et al. A Phase II Study of Bortezomib in Relapsed Refractory Multiple Myeloma. N Eng J Med 2003; 348: 2609-17. 